\title{
Extended Lorentz Transformations in Clifford Space Relativity Theory
}

\author{
Carlos Castro*
}

September, 2014

Center for Theoretical Studies of Physical Systems, Clark Atlanta University, Atlanta; perelmanc@hotmail.com

\begin{abstract}
Some novel physical consequences of the Extended Relativity Theory in $C$-spaces (Clifford spaces) were explored recently. In particular, generalized photon dispersion relations allowed for energy-dependent speeds of propagation while still retaining the Lorentz symmetry in ordinary spacetimes, but breaking the extended Lorentz symmetry in $C$-spaces. In this work we analyze in further detail the extended Lorentz transformations in Clifford Space and their physical implications. Based on the notion of "extended events" one finds a very different physical explanation of the phenomenon of "relativity of locality" than the one described by the Doubly Special Relativity (DSR) framework. A generalized Weyl-Heisenberg algebra, involving polyvector-valued coordinates and momenta operators, furnishes a realization of an extended Poincare algebra in $C$-spaces. In addition to the Planck constant $\hbar$, one finds that the commutator of the Clifford scalar components of the Weyl-Heisenberg algebra requires the introduction of a dimensionless parameter which is expressed in terms of the ratio of two length scales : the Planck and Hubble scales. We finalize by discussing the concept of "photons", null intervals, effective temporal variables and the addition/subtraction laws of generalized velocities in $C$-space.
\end{abstract}

Keywords : Clifford algebras; Extended Relativity in Clifford Spaces; Doubly Special Relativity; Quantum Clifford-Hopf algebras.

In the past years, the Extended Relativity Theory in $C$-spaces (Clifford spaces) and Clifford-Phase spaces were developed [1], [2]. The Extended Relativity theory in Cliffordspaces (C-spaces) is a natural extension of the ordinary Relativity theory whose generalized coordinates are Clifford polyvector-valued quantities which incorporate the lines, areas, volumes, and hyper-volumes degrees of freedom associated with the collective dynamics of particles, strings, membranes, p-branes (closed p-branes) moving in a D-dimensional

*Dedicated to the memory of Rachael Bowers and based on the plenary talk given at the 10-th International Conference of Clifford Algebras held in Tartu, Estonia, August 2014 
target spacetime background. C-space Relativity permits to study the dynamics of all (closed) p-branes, for different values of $\mathrm{p}$, on a unified footing.

Our theory has 2 fundamental parameters : the speed of a light $c$ and a length scale which can be set equal to the Planck length. The role of "photons" in $C$-space is played by tensionless branes. An extensive review of the Extended Relativity Theory in Clifford spaces can be found in [1]. The polyvector valued coordinates $x^{\mu}, x^{\mu_{1} \mu_{2}}, x^{\mu_{1} \mu_{2} \mu_{3}}, \ldots$ are now linked to the basis vectors generators $\gamma^{\mu}$, bi-vectors generators $\gamma_{\mu} \wedge \gamma_{\nu}$, tri-vectors generators $\gamma_{\mu_{1}} \wedge \gamma_{\mu_{2}} \wedge \gamma_{\mu_{3}}$, ... of the Clifford algebra, including the Clifford algebra unit element (associated to a scalar coordinate).

These polyvector valued coordinates can be interpreted as the quenched-degrees of freedom of an ensemble of $p$-loops associated with the dynamics of closed $p$-branes, for $p=0,1,2, \ldots, D-1$, embedded in a target $D$-dimensional spacetime background. $C$-space is parametrized not only by 1 -vector coordinates $x^{\mu}$ but also by the 2 -vector coordinates $x^{\mu \nu}, 3$-vector coordinates $x^{\mu \nu \alpha}, \ldots$, called also holographic coordinates, since they describe the holographic projections of 1-loops, 2-loops, 3-loops,..., onto the coordinate planes . By $p$-loop we mean a closed $p$-brane; in particular, a 1-loop is closed string. When $\mathbf{X}$ is the Clifford-valued coordinate corresponding to the $C l(1,3)$ algebra in four-dimensions it can be decomposed as

$$
\mathbf{X}=s \mathbf{1}+x^{\mu} \gamma_{\mu}+x^{\mu \nu} \gamma_{\mu} \wedge \gamma_{\nu}+x^{\mu \nu \rho} \gamma_{\mu} \wedge \gamma_{\nu} \wedge \gamma_{\rho}+x^{\mu \nu \rho \tau} \gamma_{\mu} \wedge \gamma_{\nu} \wedge \gamma_{\rho} \wedge \gamma_{\tau}
$$

where we have omitted combinatorial numerical factors for convenience in the expansion of eq-(1). To avoid introducing powers of a length parameter $L$ (like the Planck scale $L_{p}$ ), in order to match physical units in the expansion of the polyvector $X$ in eq-(1), we can set it to unity to simplify matters.

The component $s$ is the Clifford scalar component of the polyvector-valued coordinate and $d \Sigma$ is the infinitesimal $C$-space proper "time" interval

$$
(d \Sigma)^{2}=(d s)^{2}+d x_{\mu} d x^{\mu}+d x_{\mu \nu} d x^{\mu \nu}+\ldots
$$

that is invariant under $C l(1,3)$ transformations and which are the Clifford-algebraic extensions of the $S O(1,3)$ Lorentz transformations [1]. One should emphasize that $d \Sigma$ is not equal to the proper time Lorentz-invariant interval $d \tau$ in ordinary spacetime $(d \tau)^{2}=$ $g_{\mu \nu} d x^{\mu} d x^{\nu}=d x_{\mu} d x^{\mu}$. Generalized Lorentz transformations (poly-rotations) in flat $C$ spaces were discussed in [1]. In this work we shall provide an extensive analysis of the $C$-space generalized Lorentz transformations and their physical implications.

Let us provide several examples of generalized Lorentz transformations in $C$-space. For example, given $\gamma_{02}$ the transformation involving the rotor $R_{1}=\cosh (\beta / 2)-\gamma_{02} \sinh (\beta / 2)$ corresponds to an ordinary Lorentz boost transformation along the $X^{2}$ direction and involving the ordinary temporal variable $X^{0}$. The ordinary Lorentz boots generators are given by the bivectors $\gamma_{\mu \nu}$, and which in turn are also expressed as the commutators $\left[\gamma_{\mu}, \gamma_{\nu}\right]$. The physical significance of the latter commutators is that they represent a "rotation" along the $X^{\mu}-X^{\nu}$ directions.

However, since one may also write the bivector $\gamma_{02}$ as the commutator $\left[\gamma_{12}, \gamma_{01}\right]=$ $-2 \gamma_{02}$, the transformation involving the above rotor $R_{1}$ also corresponds to an areal boost 
along the $X^{12}$ direction but involving the areal temporal coordinate $X^{01}$. Namely, it is a "rotation" along the $X^{12}-X^{01}$ directions. Whereas the ordinary boost is a "rotation" along the $X^{2}-X^{0}$ directions.

After writing

$$
\left(X^{B}\right)^{\prime} \Gamma_{B}=\left(\cosh (\beta / 2)-\gamma_{02} \sinh (\beta / 2)\right)\left(X^{A} \Gamma_{A}\right)\left(\cosh (\beta / 2)+\gamma_{02} \sinh (\beta / 2)\right)
$$

straightforward algebra yields the transformation of the following bivector coordinates

$$
\begin{aligned}
& \left(X^{12}\right)^{\prime}=X^{12} \cosh \beta+X^{01} \sinh \beta \\
& \left(X^{01}\right)^{\prime}=X^{01} \cosh \beta+X^{12} \sinh \beta
\end{aligned}
$$

One has a mixing of the spatial and temporal areal bivector coordinates in the new frame of reference.

Furthermore, since $\left[\gamma_{013}, \gamma_{123}\right] \sim \gamma_{02}$, the transformation involving the above rotor $R_{1}$ also corresponds to a 3 -volume boost along the $X^{123}$ direction but involving the 3 -volume temporal coordinate $X^{013}$. Namely, it is a "rotation" along the $X^{123}-X^{013}$ directions giving

$$
\begin{aligned}
& \left(X^{123}\right)^{\prime}=X^{123} \cosh \beta+X^{013} \sinh \beta \\
& \left(X^{013}\right)^{\prime}=X^{013} \cosh \beta+X^{123} \sinh \beta
\end{aligned}
$$

One has a mixing of the spatial and temporal trivector coordinates in the new frame of reference. The ordinary Lorentz boosts of the vector coordinates give

$$
\begin{aligned}
& \left(X^{2}\right)^{\prime}=X^{2} \cosh \beta+X^{0} \sinh \beta \\
& \left(X^{0}\right)^{\prime}=X^{0} \cosh \beta+X^{2} \sinh \beta
\end{aligned}
$$

while the remaining coordinates remain invariant and such that the quadratic form $X^{A} X_{A}=\left(X^{A}\right)^{\prime}\left(X_{A}\right)^{\prime}$ remains invariant. Straightforward algebra leads to

$$
\begin{aligned}
& -\left(X_{0}^{\prime}\right)^{2}+\left(X_{1}^{\prime}\right)^{2}-L^{-2}\left(X_{01}^{\prime}\right)^{2}+L^{-2}\left(X_{12}^{\prime}\right)^{2}-L^{-4}\left(X_{013}^{\prime}\right)^{2}+L^{-4}\left(X_{123}^{\prime}\right)^{2}= \\
& -\left(X_{0}\right)^{2}+\left(X_{1}\right)^{2}-L^{-2}\left(X_{01}\right)^{2}+L^{-2}\left(X_{12}\right)^{2}-L^{-4}\left(X_{013}\right)^{2}+L^{-4}\left(X_{123}\right)^{2}
\end{aligned}
$$

The quadratic form is defined as

$$
<\mathbf{X}^{\dagger} \mathbf{X}>=X_{A} X^{A}=s^{2}+X_{\mu} X^{\mu}+X_{\mu_{1} \mu_{2}} X^{\mu_{1} \mu_{2}}+\ldots \ldots X_{\mu_{1} \mu_{2} \ldots . . \mu_{D}} X^{\mu_{1} \mu_{2} \ldots \mu_{D}}
$$

where $\mathbf{X}^{\dagger}$ denotes the reversal operation obtained by reversing the order of the gamma generators in the wedge products. The symbol $<\Gamma_{A} \Gamma_{B}>$ denotes taking the scalar part in the Clifford geometric product of $\Gamma_{A} \Gamma_{B}$. It is the analog of the trace of a product of matrices. Such scalar part can be obtained from the (anti) commutator relations of the Clifford algebra generators as displayed in the Appendix. For example 


$$
\begin{gathered}
<\gamma_{\mu} \gamma^{\nu}>=\delta_{\mu}^{\nu}, \quad<\gamma_{\mu_{1} \mu_{2}} \gamma^{\nu_{1} \nu_{2}}>=-\delta_{\mu_{1} \mu_{2}}^{\nu_{1} \nu_{2}} \\
<\gamma_{\mu_{1} \mu_{2} \mu_{3}} \gamma^{\nu_{1} \nu_{2} \nu_{3}}>=-\delta_{\mu_{1} \mu_{2} \mu_{3} \nu_{3}}^{\nu_{1} \nu_{3}}, \quad<\gamma_{\mu_{1} \mu_{2} \mu_{3} \mu_{4}} \gamma^{\nu_{1} \nu_{2} \nu_{3} \nu_{4}}>=\delta_{\mu_{1} \mu_{2} \mu_{3} \mu_{4} \nu_{4}}^{\nu_{2} \nu_{4}},
\end{gathered}
$$

One should note the presence of \pm signs in the right hand side of eqs-(9). They are connected to the even/odd behavior of the reversal operation $\left(\gamma_{C}\right)^{\dagger}= \pm \gamma_{C}$.

The quadratic form is invariant under the isometry transformations

$$
\mathbf{X}^{\prime}=\mathbf{R} \mathbf{X ~ L}^{\dagger}, \quad \mathbf{R} \mathbf{R}^{\dagger}=1, \quad \mathbf{L ~}^{\dagger}=1 \Rightarrow<\mathbf{X}^{\prime \dagger} \mathbf{X}^{\prime}>=<\mathbf{X}^{\dagger} \mathbf{X}>
$$

due to the cyclic property of the scalar part projection

$$
\begin{aligned}
<\mathbf{X}^{\prime \dagger} \mathbf{X}^{\prime}>= & <\mathbf{L} \mathbf{X}^{\dagger} \mathbf{R}^{\dagger} \mathbf{R} \mathbf{X} \mathbf{L}^{\dagger},>=<\mathbf{L} \mathbf{X}^{\dagger} \mathbf{X} \mathbf{L}^{\dagger}>= \\
& <\mathbf{L}^{\dagger} \mathbf{L} \mathbf{X}^{\dagger} \mathbf{X}=<\mathbf{X}^{\dagger} \mathbf{X}>
\end{aligned}
$$

where $\mathbf{R}, \mathbf{L}$ are Clifford-valued rotors acting on the right and left respectively.

The second example corresponds to the case when there is a mixing of different grades. It involves the commutator $\left[\gamma_{0123}, \gamma_{3}\right] \sim \gamma_{012}$ and such that the transformation involving the rotor $R_{2}=\cosh \left(\beta^{\prime} / 2\right)-\gamma_{012} \sinh \left(\beta^{\prime} / 2\right)$ corresponds to a boost along the spatial $X^{3}$ direction but involving now the temporal 4 -volume polyvector-valued coordinate $X^{0123}$. The reason being that $\gamma_{012}$ can be rewritten as the commutator of $\gamma_{0123}$ and $\gamma_{3}$, so we have now "rotations" along the $X^{3}-X^{0123}$ directions. Straightforward algebra yields now the transformation of the following (poly) vector coordinates

$$
\begin{aligned}
& \left(X^{3}\right)^{\prime}=X^{3} \cosh \left(\beta^{\prime}\right)-L^{-3} X^{0123} \sinh \left(\beta^{\prime}\right) \\
& \left(X^{0123}\right)^{\prime}=X^{0123} \cosh \left(\beta^{\prime}\right)-L^{3} X^{3} \sinh \left(\beta^{\prime}\right)
\end{aligned}
$$

In this case one has a mixing of polyvector-valued coordinates of different grade. In the new frame of reference the spatial $X^{3}$ coordinate and the temporal 4 -volume coordinate $X^{0123}$ are mixed.

Furthermore, since $\left[\gamma_{03}, \gamma_{123}\right] \sim \gamma_{012}$, the transformation involving the rotor $R_{2}=$ $\cosh \left(\beta^{\prime} / 2\right)-\gamma_{012} \sinh \left(\beta^{\prime} / 2\right)$ also corresponds to a boost along the spatial trivector $X^{123}$ direction but involving now the temporal bivector coordinate $X^{03}$. These transformations are

$$
\begin{gathered}
\left(X^{123}\right)^{\prime}=X^{123} \cosh \left(\beta^{\prime}\right)-L X^{03} \sinh \left(\beta^{\prime}\right) \\
\left(X^{03}\right)^{\prime}=X^{03} \cosh \left(\beta^{\prime}\right)-L^{-1} X^{123} \sinh \left(\beta^{\prime}\right)
\end{gathered}
$$

In the above equations we have used the relations

$$
\begin{gathered}
\gamma_{01}^{2}=1, \quad \gamma_{02}^{\dagger}=-\gamma_{02}, \quad \gamma_{012}^{2}=1, \quad \gamma_{012}^{\dagger}=-\gamma_{012} \\
\left\{\gamma_{12}, \gamma_{02}\right\}=0, \quad\left[\gamma_{0123}, \gamma_{012}\right]=-2 \gamma_{3}, \quad\left\{\gamma_{0123}, \gamma_{012}\right\}=0
\end{gathered}
$$




$$
\gamma_{02} \gamma_{12} \gamma_{02}=-\gamma_{12}, \quad\left[\gamma_{012}, \gamma_{3}\right]=2 \gamma_{0123}, \quad\left\{\gamma_{012}, \gamma_{3}\right\}=0, \ldots
$$

$\cosh ^{2}(\xi)-\sinh ^{2}(\xi)=1, \cosh ^{2}(\xi)+\sinh ^{2}(\xi)=\cosh (2 \xi), \sinh (2 \xi)=2 \sinh (\xi) \cosh (\xi)$

Given in general a transformation of the form

$$
\left(\cosh (\beta / 2)-\Gamma_{C} \sinh (\beta / 2)\right) X^{A} \Gamma_{A}\left(\cosh (\beta / 2)+\Gamma_{C} \sinh (\beta / 2)\right)=X^{\prime B} \Gamma_{B}
$$

one learns that

$$
\begin{gathered}
X^{\prime B}=X^{B} \cosh ^{2}(\beta / 2)-X^{A} \sinh ^{2}(\beta / 2)<\Gamma_{C} \Gamma_{A} \Gamma_{C} \Gamma^{B}>+ \\
X^{A} \cosh (\beta / 2) \sinh (\beta / 2)<\left[\Gamma_{A}, \Gamma_{C}\right] \Gamma^{B}>
\end{gathered}
$$

The generator $\Gamma_{C}$ of generalized Lorentz boosts is of the form $\left(\gamma_{0 \mu_{1} \mu_{2} \ldots \mu_{n-1}}\right)$ with the provision that under the reversal operation it changes sign

$$
\left(\gamma_{0 \mu_{1} \mu_{2} \ldots \mu_{n-1}}\right)^{\dagger}=-\gamma_{0 \mu_{1} \mu_{2} \ldots \mu_{n-1}}
$$

so that $\mathbf{R R}^{\dagger}=1$. This condition will restrict the values of $n$ to be $n=2,3,6$, ... and obeying

$$
\left(\gamma_{0 \mu_{1} \mu_{2} \ldots \mu_{n-1}}\right)^{2}=1
$$

Generalized spatial rotations don't involve the temporal directions and are generated by $\gamma_{\mu_{1} \mu_{2} \ldots \mu_{m}}$ obeying

$$
\left(\gamma_{\mu_{1} \mu_{2} \ldots \mu_{m}}\right)^{\dagger}=-\gamma_{\mu_{1} \mu_{2} \ldots \mu_{m}}
$$

and

$$
\left(\gamma_{\mu_{1} \mu_{2} \ldots \mu_{m}}\right)^{2}=-1
$$

For instance, a generalized rotation in $D>4$ and generated by $\gamma_{12 \ldots 6}$ involving the parameter $\alpha^{12 \ldots 6}$ yields a rotor whose Taylor series expansion becomes

$$
\mathbf{R}=e^{\alpha^{12 \ldots 6}} \gamma_{12 \ldots . .6}=\cos \left(\alpha^{12 \ldots 6}\right)+\gamma_{012 \ldots .6} \sin \left(\alpha^{12 \ldots 6}\right)
$$

due to the condition $\left(\gamma_{12 \ldots . .6}\right)^{2}=-1$ which is similar to having the imaginary unit $i^{2}=-1$ and the expression $e^{i \theta}=\cos (\theta)+i \sin (\theta)$. Whereas a generalized Lorentz boost is like having a "rotation" with an imaginary "angle" leading to the hyperbolic functions

$$
\mathbf{R}=e^{\beta^{012 \ldots 5}} \gamma_{02 \ldots . .5}=\cosh \left(\beta^{012 \ldots .5}\right)+\gamma_{012 \ldots .5} \sinh \left(\beta^{12 \ldots 5}\right)
$$

due to the condition $\left(\gamma_{012 \ldots . .5}\right)^{2}=1$.

Eq-(17) only simplifies considerably in the very special case when the values of the polyvector valued indices $A, B, C$ are such that 


$$
<\Gamma_{C} \Gamma_{A} \Gamma_{C} \Gamma^{B}>=-1, \quad<\left[\Gamma_{A}, \Gamma_{C}\right] \Gamma^{B}>= \pm 2
$$

and it leads to the type of transformations displayed above. In general, for a given set of values of $B, C$, one must sum over all the $A$ indices in eq-(17). For this reason the most general expression for $X^{\prime B}$ given by eq-(17) is more complicated than that given by the above equations. Another special case occurs when

$$
<\Gamma_{C} \Gamma_{A} \Gamma_{C} \Gamma^{B}>=1, \quad<\left[\Gamma_{A}, \Gamma_{C}\right] \Gamma^{B}>=0
$$

leading to $X^{\prime B}=X^{B}$ so that these particular polyvector coordinate components remain invariant.

One should emphasize that the functional form of the most general transformations are even more complicated than those described in eq-(17). Let us write the rotor associated with a "rotation" along the $X^{A}-X^{B}$ directions in $C$-space with parameter $\alpha^{A B}$, after writing the commutation relations $\left[\Gamma_{A}, \Gamma_{B}\right]=f_{A B} \Gamma_{C}$, as follows

$$
\mathbf{R}=e^{\alpha^{A B}\left[\Gamma_{A}, \Gamma_{B}\right]}=e^{\alpha^{A B}} f_{A B}^{C} \Gamma_{C}=e^{\beta^{C} \Gamma_{C}}, \quad \beta^{C}=\alpha^{A B} f_{A B} C
$$

where $f_{A B} C$ are the structure constants of the algebra. There is a summation over the $C$ indices (but not over the $A, B$ indices) in eq-(25) and the reversal condition reads

$$
\left[\Gamma_{A}, \Gamma_{B}\right]^{\dagger}=-\left[\Gamma_{A}, \Gamma_{B}\right] \Rightarrow \mathbf{R} \mathbf{R}^{\dagger}=1
$$

and which is satisfied in particular when $\Gamma_{A}^{\dagger}=-\Gamma_{A} ; \Gamma_{B}^{\dagger}=-\Gamma_{B}$ giving $\Gamma_{C}^{\dagger}=-\Gamma_{C}$. This is a result of the relations $\left(\Gamma_{A} \Gamma_{B}\right)^{\dagger}=\left(\Gamma_{B}\right)^{\dagger}\left(\Gamma_{A}\right)^{\dagger}=\Gamma_{B} \Gamma_{A}$. In the most general case, for arbitrary dimensions, due to the summation over the $C$ polyvector indices in eq- $(25)$, the rotor $\mathbf{R}$ cannot be expressed in the form displayed in eq-(16) after performing a Taylor series expansion of the exponentials. For instance

$$
e^{\beta^{01} \gamma_{01}+\beta^{023} \gamma_{023}} \neq\left(\cosh \left(\beta^{01}\right)+\gamma_{01} \sinh \left(\beta^{01}\right)\right)\left(\cosh \left(\beta^{023}\right)+\gamma_{023} \sinh \left(\beta^{023}\right)\right)
$$

as a result of the Baker-Campbell-Hausdorf formula. Because $\left[\gamma_{01}, \gamma_{023}\right] \neq 0$ the left hand side of eq-(27) does not factorize.

We shall study next another different approach to the construction of generalized Lorentz transformations involving only polyvector components of equal grade. One may define a generalized Lorentz algebra in terms of anti-Hermitian operators $\mathcal{J}^{A B}=-\mathcal{J}^{B A}$ as $^{1}$

$$
\left[\mathcal{J}^{A B}, \mathcal{J}^{C D}\right]=-G^{A C} \mathcal{J}^{B D}+G^{A D} \mathcal{J}^{B C}-G^{B D} \mathcal{J}^{A C}+G^{B C} \mathcal{J}^{A D}
$$

where $A, B, C, \ldots$ are polyvector-valued indices. One must emphasize that $\mathcal{J}^{A B} \neq$ $\left[\Gamma^{A}, \Gamma^{B}\right]$, except in the case $\mathcal{J}^{\mu \nu}=\frac{1}{4}\left[\gamma^{\mu}, \gamma^{\nu}\right]$. To simplify matters, the generalized metric

\footnotetext{
${ }^{1}$ We choose anti-Hermitian operators in order to avoid having to introduce $\mathbf{i}$ factors in the right hand side of the commutators
} 
$G^{A B}=G^{B A}$ shall be chosen to be $G^{A B}=0$ when the grade $A \neq$ grade $B$. And for the same grade metric components $g^{\left[a_{1} a_{2} \ldots a_{k}\right]\left[b_{1} b_{2} \ldots b_{k}\right]}$ of $G^{A B}$, the metric can decomposed into its irreducible factors as antisymmetrized sums of products of $\eta^{a b}$ given by the following determinant [8]

$$
G^{A B} \equiv \operatorname{det}\left(\begin{array}{ccc}
\eta^{a_{1} b_{1}} & \ldots & \ldots \eta^{a_{1} b_{k}} \\
\eta^{a_{2} b_{1}} & \ldots & \ldots \eta^{a_{2} b_{k}} \\
---\frac{-1}{\eta^{a_{k} b_{1}}} & \ldots & \ldots \eta^{a_{k} b_{k}}
\end{array}\right)=G^{B A}
$$

The spacetime signature is chosen to be $(-,+,+, \ldots,+)$.

One can verify next that a realization of the algebra (28) can be obtained in terms of polyvector-valued coordinates and momenta $\hat{X}^{A}, \hat{P}^{B}$ operators obeying the generalized Weyl-Heisenberg algebra

$$
\left[\hat{X}^{A}, \hat{P}^{B}\right]=i(\hbar)^{(|A|+|B|) / 2} G^{A B}, \quad G^{A B}=G^{B A}
$$

where $|A|,|B|,=$ grade of $A, B$, respectively.

The $C$-space polyvector-valued momentum is defined as

$$
\mathbf{P}=\mathcal{M} \frac{d \mathbf{X}}{d \Sigma}=P^{A} \Gamma_{A}=\pi+p^{\mu} \gamma_{\mu}+p^{\mu \nu} \gamma_{\mu} \wedge \gamma_{\nu}+\ldots \ldots
$$

where $(d \Sigma)^{2}=<d \mathbf{X}^{\dagger} d \mathbf{X}>$. $\quad \Sigma$ is the analog of "proper time" in $C$-space. To match physical units, powers of a suitable mass/length parameter must be introduced in eq-(31). Like the Planck mass and length. If $\mathbf{X}$ and $\mathbf{P}$ are taken to have length and momentum dimensions, respectively, then $\mathcal{M}$ has mass dimensions. By inspection one learns that the commutator of the zero grade components, the scalar parts of $\hat{X}^{A}$ and $\hat{P}^{B}$, does not involve $\hbar$ but a dimensionless parameter that can be given by the ratio of an ultraviolet $L_{P}$ and infrared Hubble scale $R_{H}$ as follows

$$
[\hat{s}, \hat{\pi}]=i \frac{L_{P}}{R_{H}} G^{* *}
$$

$G^{* *}$ is the scalar-scalar component of the generalized metric $G^{A B}$. The classical limit is attained when $L_{P} / R_{H} \rightarrow 0$ so that the above commutator vanishes. This ratio $L_{P} / R_{H}$ is also related to the observed vacuum energy density $\rho \sim\left(L_{P} / R_{H}\right)^{2}\left(M_{P}\right)^{4} \sim$ $10^{-122}$ (Planck Mass) ${ }^{4}$.

Hence, if $\mathcal{J}^{A B}=0$ when the grade $A \neq$ grade $B$, a Weyl-Heisenberg algebra allows to find a realization of the dimensionless anti-Hermitian generators $\mathcal{J}^{A B}$ in eq-(28) as follows

$$
\mathcal{J}^{A B}=\frac{i}{\hbar^{(|A|+|B|) / 2}}\left(\hat{X}^{A} \hat{P}^{B}-\hat{X}^{B} \hat{P}^{A}\right)=-\mathcal{J}^{B A}, \quad \mathcal{J}^{A B}=0 \text { if }|A| \neq|B|
$$

$\hat{X}^{A}$ and $\hat{P}^{B}$ are Hermitian operators. 
To sum up, when $|A|=|B|, G^{A B} \neq 0, \mathcal{J}^{A B} \neq 0$; and $G^{A B}=0, \mathcal{J}^{A B}=0$ for $|A| \neq|B|$, a generalization of the Poincare algebra involving polyvector-valued indices is given by the commutators in eq- $(28)$ and

$$
\left[\mathcal{J}^{A B}, \hat{P}^{C}\right]=-G^{A C} \hat{P}^{B}+G^{B C} \hat{P}^{A}, \quad\left[\hat{P}^{A}, \hat{P}^{B}\right]=0,\left[\hat{X}^{A}, \hat{X}^{B}\right]=0,
$$

where $\hat{P}^{A}$ are the polymomentum operators and $\mathcal{J}^{A B}$ are the generalized Lorentz generators. The $\left[\mathcal{J}^{A B}, \mathcal{J}^{C D}\right],\left[\mathcal{J}^{A B}, \hat{P}^{C}\right], \ldots$ commutators obey the Jacobi identities.

A question still remains whether or not it is possible to construct the generators of the algebra displayed by eq-(28) in terms of a judicious superposition of Clifford algebra generators like

$$
\mathcal{J}^{A B}=M_{C}^{A B} \Gamma^{C}
$$

By inspection one learns that $\mathcal{J}^{A B} \neq\left[\Gamma^{A}, \Gamma^{B}\right]$, nor proportional to the commutators, except in the case $\mathcal{J}^{\mu \nu}=\frac{1}{4}\left[\gamma^{\mu}, \gamma^{\nu}\right]$. Therefore, the coefficients $M_{C}^{A B} \neq f_{C}^{A B}$ are not given by the structure constants. Inserting the ansatz of (35) into the commutators (28) leads to an algebraic set of equations involving $M_{C}^{A B}, f_{C}^{A B}, G^{A B}$ as the indices $A, B, C$ run from 1 to $2^{D}$. It is unknown (to our knowledge) if a solution for the coefficients $M_{C}^{A B}$ exists given the complexity of the (anti) commutator relations in any dimension provided in the Appendix. A computer algebra package would be required.

We learnt from Special Relativity that the concept of simultaneity is relative. The typical example arises when a moving observer inside a train sees the front and back doors of a train opening simultaneously. Due to the spatial separation $\left(\Delta X^{3} \neq 0\right)$ between the two doors, an observer at rest in the platform will see the doors opening at different times

$$
\left(\Delta X^{0}\right)^{\prime}=\Delta X^{0} \cosh (\beta)+\Delta X^{3} \sinh (\beta) \neq 0,
$$

despite $\Delta X^{0}=0$ due to the fact that $\Delta X^{3} \neq 0$.

Something analogous, and more general, occurs in $C$-space. Let us denote by $\Delta X^{3}=$ $X_{(2)}^{3}-X_{(1)}^{3}, \Delta X^{0123}=X_{(2)}^{0123}-X_{(1)}^{0123}$ the spatial and 4-volume separation, respectively, between two events 1 and $\mathbf{2}$ in a given frame of reference in a flat $C$-space. From eqs-(12) it follows that in the new frame of reference one has

$$
\begin{aligned}
& \left(\Delta X^{3}\right)^{\prime}=\Delta X^{3} \cosh \left(\beta^{\prime}\right)-L^{-3} \Delta X^{0123} \sinh \left(\beta^{\prime}\right) \\
& \left(\Delta X^{0123}\right)^{\prime}=\Delta X^{0123} \cosh \left(\beta^{\prime}\right)-L^{3} \Delta X^{3} \sinh \left(\beta^{\prime}\right)
\end{aligned}
$$

if $\Delta X^{0123} \neq 0$ one has that $\left(\Delta X^{3}\right)^{\prime} \neq 0$ despite that $\Delta X^{3}=0$. Therefore, because $\left(\Delta X^{3}\right)^{\prime} \neq 0$ the observer in the new frame of reference does not experience events $\mathbf{1}, \mathbf{2}$ at the same location.

An "extended" event in $C$-space described by eqs-(37) can be envisaged as follows. An observer assigns to a physical event the coordinate values $X^{A}$ where the index $A$ spans $2^{D}$ values corresponding to the dimension of a Clifford algebra in $D$-dim. In particular $X^{3}, X^{0123}$. Event 1 can be described in terms of a spherical bubble (a closed 3-brane) moving in spacetime whose 4 -volume (swept by the 3 -brane at a given time $X_{(1)}^{0}$ ) is given by $X_{(1)}^{0123}$. The center of mass of such bubble is given by the $X_{(1)}^{\mu}$ coordinates, in 
particular $X_{(1)}^{3}$ represents the $z$-component. Whereas event $\mathbf{2}$ is described in terms of another spherical bubble of different size in spacetime whose 4-volume at a given time $X_{(2)}^{0}$ is given by $X_{(2)}^{0123}$. The center of mass of such bubble is given now by $X_{(2)}^{\mu}$ coordinates, in particular $X_{(2)}^{3}$. If the centers of mass of the small and large bubble coincide one has that $\Delta X^{3}=0$, while $\Delta X^{0123} \neq 0$ since the bubbles are of different size. Consequently one learns from eq- $(37 \mathrm{a})$ that $\left(\Delta X^{3}\right)^{\prime} \neq 0$ in the new frame of reference : namely, the centers of mass of the bubbles in the new frame of reference do no longer coincide.

Concluding, the concept of spacetime locality is relative due to the mixing of 4-volume coordinates with spacetime vector coordinates under generalized Lorentz transformations in $C$-space. In the most general case, there will be mixing of all polyvector valued coordinates. This was the motivation to build a unified theory of all extended objects, $p$-branes, for all values of $p$ subject to the condition $p+1=D$. Therefore, the Extended Relativity Theory in $C$-spaces (Clifford spaces) were provides a very different physical explanation of the phenomenon of "relativity of locality" than the one described by the Doubly Special Relativity (DSR) framework [7].

Recently it was found in [4] that the constant (energy-independent) speed of photon propagation is always compatible with the generalized photon dispersion relations in $C$-spaces. Another important consequence was that the generalized $C$-space photon dispersion relations allowed also for energy-dependent speeds of propagation while still retaining the Lorentz symmetry in ordinary spacetimes, while breaking the extended Lorentz symmetry in $C$-spaces. This does not occur in DSR nor in other approaches, like the presence of quantum spacetime foam.

To finalize we shall discuss the concept of "photons" and generalized velocities in $C$-space. Superluminal particles were studied within the framework of the Extended Relativity theory in Clifford spaces (C-spaces) in [5]. As discussed in detailed by [1], [3] one can have tachyonic (superluminal) behavior in ordinary spacetime while having nontachyonic behavior in $C$-space. Hence from the $C$-space point of view there is no violation of causality nor the Clifford-extended Lorentz symmetry. The analog of "photons" in $C$ space are tensionless strings and branes [1].

Let us take the spacetime signature to be $(-,+,+,+, \ldots \ldots,+)$ and factorize the $C$ space interval in eq- $(2)$ as follows by bringing the $c^{2}(d t)^{2}$ factor outside the parenthesis

$(d \Sigma)^{2}=c^{2}(d t)^{2}\left(\frac{L^{2}}{c^{2}}\left(\frac{d s}{d t}\right)^{2}-1+\frac{1}{c^{2}}\left(\frac{d X_{i}}{d t}\right)^{2}+\frac{1}{L^{2} c^{2}}\left(\frac{d X_{i j}}{d t}\right)^{2}-\frac{1}{L^{2} c^{2}}\left(\frac{d X_{0 i}}{d t}\right)^{2} \ldots \ldots ..\right)$

where the spatial index $i$ range is $1,2, \ldots, D-1$. The Clifford space associated with the Clifford algebra in $4 D$ is 16-dimensional and has a neutral/split signature of $(8,8)[3],[1]$. For example, the terms $\left(d X_{0 i}\right)^{2},\left(d X_{0 i j}\right)^{2},\left(d X_{0123}\right)^{2}$ will appear with a negative sign, while the terms $\left(d X_{i j}\right)^{2},\left(d X_{i j k}\right)^{2}$ will appear with a positive sign.

There are many possible combination of numerical values for the 16 terms inside the parenthesis in eq-(27). As explained in [3], [1], superluminal velocities in ordinary spacetime are possible, while retaining the null interval condition in $C$-space $(d \Sigma)^{2}=0$, associated with tensionless branes. The null interval in $C$-space $(d \Sigma)^{2}=0$ can be 
attained, for example, if each term inside the parenthesis is \pm 1 respectively. Since there are 8 positive $(+1)$ terms and 8 negative $(-1)$ terms one has that $8-8=0$ and the null interval condition $(d \Sigma)^{2}=0$ is still satisfied despite having superluminal speeds.

A very different combination of numerical values, as compared to the previous one, leading also to a null interval condition in $C$-space $(d \Sigma)^{2}=0$, occurs when

$$
\begin{gathered}
\frac{1}{c^{2}}\left(\left(\frac{d X_{1}}{d t}\right)^{2}+\left(\frac{d X_{2}}{d t}\right)^{2}+\left(\frac{d X_{3}}{d t}\right)^{2}\right)=1 \\
\frac{1}{L^{2} c^{2}}\left(\left(\frac{d X_{12}}{d t}\right)^{2}+\left(\frac{d X_{13}}{d t}\right)^{2}+\left(\frac{d X_{23}}{d t}\right)^{2}\right)= \\
\frac{1}{L^{2} c^{2}}\left(\left(\frac{d X_{01}}{d t}\right)^{2}+\left(\frac{d X_{02}}{d t}\right)^{2}+\left(\frac{d X_{03}}{d t}\right)^{2}\right) \\
\frac{1}{L^{4} c^{2}}\left(\left(\frac{d X_{012}}{d t}\right)^{2}+\left(\frac{d X_{013}}{d t}\right)^{2}+\left(\frac{d X_{023}}{d t}\right)^{2}\right)=\frac{1}{L^{4} c^{2}}\left(\frac{d X_{123}}{d t}\right)^{2} \\
\frac{1}{L^{6} c^{2}}\left(\frac{d X_{0123}}{d t}\right)^{2}=\frac{L^{2}}{c^{2}}\left(\frac{d s}{d t}\right)^{2}
\end{gathered}
$$

Another description of $C$-space "photons" can then be given in terms of an ef fective temporal variable $T$ comprised of all the temporal coordinates in the interval of eq- $(38)$. In order to simplify matters let us work with $D=3$ instead of $D=4$. The effective temporal variable $T$ is defined as

$$
c^{2}(d T)^{2} \equiv c^{2}(d t)^{2}+\frac{1}{c^{2}}\left(\frac{d X_{01}}{d t}\right)^{2}+\frac{1}{c^{2}}\left(\frac{d X_{02}}{d t}\right)^{2}+\frac{1}{L^{2} c^{2}}\left(\frac{d X_{012}}{d t}\right)^{2}
$$

so that the $C$-space interval can be rewritten, after factoring out the $c^{2}(d T)^{2}$ term, as

$$
(d \Sigma)^{2}=-c^{2}(d T)^{2}\left(1-\frac{L^{2}}{c^{2}}\left(\frac{d s}{d T}\right)^{2}-\frac{1}{c^{2}}\left(\frac{d X_{1}}{d T}\right)^{2}-\frac{1}{c^{2}}\left(\frac{d X_{2}}{d T}\right)^{2}-\frac{1}{L^{2} c^{2}}\left(\frac{d X_{12}}{d T}\right)^{2}\right)
$$

The last expression has the same functional form as the ordinary spacetime interval in MInkowski space. Namely one can write the $C$-space interval $(d \Sigma)^{2}$ in the form

$$
(d \Sigma)^{2}=-c^{2}(d T)^{2}\left(1-\frac{V^{2}}{c^{2}}\right)
$$

where the generalization of the magnitude-squared of the spatial velocity divided by $c^{2}$ is

$$
\frac{V^{2}}{c^{2}} \equiv \frac{L^{2}}{c^{2}}\left(\frac{d s}{d T}\right)^{2}+\frac{1}{c^{2}}\left(\frac{d X_{1}}{d T}\right)^{2}+\frac{1}{c^{2}}\left(\frac{d X_{2}}{d T}\right)^{2}+\frac{1}{L^{2} c^{2}}\left(\frac{d X_{12}}{d T}\right)^{2}
$$

Another description of $\mathrm{C}$-space Photons is obtained from the null $C$-space interval condition $(d \Sigma)^{2}=0$ which is equivalent to setting $V^{2} / c^{2}=1$ in eq-(43) and where the velocity squared is defined with respect to the effective temporal variable $T$. 
To finalize let us write down the addition law of generalized velocities based on the extended Lorentz transformations described in this work. Upon defining $\beta=-\beta^{\prime}$ in eqs-(12) and differentiating gives

$$
\begin{gathered}
d X_{3}^{\prime}=d X_{3} \cosh \beta+L^{-3} d X_{0123} \sinh \beta \\
d X_{0123}^{\prime}=d X_{0123} \cosh \beta+L^{3} d X_{3} \sinh \beta
\end{gathered}
$$

such that

$$
\frac{d X_{3}^{\prime}}{d X_{0123}^{\prime}}=\frac{\frac{d X_{3}}{d X_{0123}}+L^{-3} \tanh \beta}{1+L^{3} \frac{d X_{3}}{d X_{0123}} \tanh \beta}
$$

Using the following definitions of the generalized velocities (in $c=1$ units)

$$
V_{3} \equiv \frac{d X_{3}}{d X_{0123}}, \quad V_{3}^{\prime \prime} \equiv L^{-3} \tanh \beta,
$$

corresponding, respectively, to the generalized velocity $V_{3}$ of a polyparticle with respect to the temporal 4-volume $X^{0123}$ coordinate (as measured in a given frame of reference) and the generalized velocity $V_{3}^{\prime \prime}$ of a moving observer associated with the generalized boost transformation with parameter $\beta$. Hence, eq-(46) can be rewritten as

$$
V_{3}^{\prime}=\frac{V_{3}+V_{3}^{\prime \prime}}{1+\frac{V_{3} V_{3}^{\prime}}{L^{-6}}}
$$

leading to the addition law of the generalized velocities. In particular, one can see that if the maximal generalized velocity is identified with the quantity $c L^{-3}$, after restoring the speed of light that was set to unity, we have that the addition/subtraction law of the maximal generalized velocities $c L^{-3}$ yields always the maximal generalized velocity

$$
V_{3}^{\prime}=\frac{V_{3} \pm V_{3}^{\prime \prime}}{1 \pm \frac{V_{3} V_{3}^{\prime \prime}}{L^{-6} c^{2}}}=\frac{L^{-3} c \pm L^{-3} c}{1 \pm \frac{L^{-3} c L^{-3} c}{L^{-6} c^{2}}}=L^{-3} c \frac{1 \pm 1}{1 \pm 1}=L^{-3} c
$$

so that the maximal velocity $c L^{-3}$ is never surpassed and it is a $C$-space relativistic invariant quantity. Meaning also that if the velocities of two polyparticles in a given reference frame is maximal $c L^{-3}$, their relative velocity is also maximal resulting from the subtraction law in eq-(48).

Following the same procedure in eqs-(13) as performed above one arrives at

$$
V_{123}^{\prime}=\frac{V_{123}+V_{123}^{\prime \prime}}{1+\frac{V_{1233} V_{1233}^{\prime}}{L^{2} c^{2}}}, \quad V_{123}=c \frac{d X_{123}}{d X_{03}}, \quad V_{123}^{\prime \prime}=c L \tanh (\beta), \quad V_{123}^{\prime}=c \frac{d X_{123}^{\prime}}{d X_{03}^{\prime}}
$$

where the maximal generalized velocity $V_{123}$ is now $c L$. In general, the maximal values of the generalized velocities are $c$ and $c L^{n}$ where $n$ is a positive, negative integer. The case $n=0$ corresponds to a generalized velocity associated with polyvector-valued coordinates 
of the same grade ${ }^{2}$. Namely, $c\left(d X^{\mu_{1} \mu_{2} \ldots \mu_{n}} / d X^{0 \nu_{1} \nu_{2} \ldots \nu_{n-1}}\right)$ such that the maximal velocity is the speed of light. More research is warranted to explore many more novel consequences of Clifford Space Relativity. Progress in the construction of generalized gravitational theories in Clifford spaces can be found in [8]. We must remark that one has not been trying to "squeeze" new physics out of Clifford algebras in this work. One the contrary, it is the physics of $p$-branes that led us to Clifford space relativity in the first place.

\section{APPENDIX}

In this Appendix we shall write the (anti) commutator relations for the Clifford algebra generators.

$$
\begin{gathered}
\frac{1}{2}\left\{\gamma_{a}, \gamma_{b}\right\}=g_{a b} \mathbf{1} ; \frac{1}{2}\left[\gamma_{a}, \gamma_{b}\right]=\gamma_{a b}=-\gamma_{b a}, a, b=1,2,3, \cdots, m \\
{\left[\gamma_{a}, \gamma_{b c}\right]=2 g_{a b} \gamma_{c}-2 g_{a c} \gamma_{b}, \quad\left\{\gamma_{a}, \gamma_{b c}\right\}=2 \gamma_{a b c}} \\
{\left[\gamma_{a b}, \gamma_{c d}\right]=-2 g_{a c} \gamma_{b d}+2 g_{a d} \gamma_{b c}-2 g_{b d} \gamma_{a c}+2 g_{b c} \gamma_{a d}}
\end{gathered}
$$

In general one has [6]

$$
\begin{aligned}
& p q=\mathbf{o d d}, \quad\left[\gamma_{m_{1} m_{2} \ldots m_{p}}, \gamma^{n_{1} n_{2} \ldots n_{q}}\right]=2 \gamma_{m_{1} m_{2} \ldots m_{p}}^{n_{1} n_{2} \ldots n_{q}}-\frac{2 p ! q !}{2 !(p-2) !(q-2) !} \delta_{\left[m_{1} m_{2}\right.}^{\left[n_{1} n_{2}\right.} \gamma_{\left.m_{3} \ldots \ldots m_{p}\right]}^{\left.n_{3} \ldots n_{q}\right]}+ \\
& \frac{2 p ! q !}{4 !(p-4) !(q-4) !} \delta_{\left[m_{1} \ldots m_{4}\right.}^{\left[n_{1} \ldots n_{4}\right.} \gamma_{\left.m_{5} \ldots \ldots m_{p}\right]}^{\left.n_{5} \ldots n_{q}\right]}-\ldots \ldots \ldots \ldots \\
& p q=\text { even, }\left\{\gamma_{m_{1} m_{2} \ldots m_{p}}, \gamma^{n_{1} n_{2} \ldots . n_{q}}\right\}=2 \gamma_{m_{1} m_{2} \ldots m_{p}}^{n_{1} n_{2} \ldots n_{q}}-\frac{2 p ! q !}{2 !(p-2) !(q-2) !} \delta_{\left[m_{1} m_{2}\right.}^{\left[n_{1} n_{2}\right.} \gamma_{\left.m_{3} \ldots \ldots m_{p}\right]}^{\left.n_{3} \ldots n_{q}\right]}+ \\
& \frac{2 p ! q !}{4 !(p-4) !(q-4) !} \delta_{\left[m_{1} \ldots m_{4}\right.}^{\left[n_{1} \ldots n_{4}\right.} \gamma_{\left.m_{5} \ldots \ldots m_{p}\right]}^{\left.n_{5} \ldots . n_{q}\right]}-\ldots \ldots \ldots \ldots \\
& p q=\text { even, } \quad\left[\gamma_{m_{1} m_{2} \ldots m_{p}}, \gamma^{n_{1} n_{2} \ldots . n_{q}}\right]=\frac{(-1)^{p-1} 2 p ! q !}{1 !(p-1) !(q-1) !} \delta_{\left[m_{1}\right.}^{\left[n_{1}\right.} \gamma_{\left.m_{2} \ldots m_{p}\right]}^{\left.n_{2} \ldots n_{q}\right]}- \\
& \frac{(-1)^{p-1} 2 p ! q !}{3 !(p-3) !(q-3) !} \delta_{\left[m_{1} m_{2} m_{3}\right.}^{\left[n_{1} n_{2} n_{3}\right.} \gamma_{\left.m_{4} \ldots \ldots m_{p}\right]}^{\left.n_{4} \ldots . n_{q}\right]}+\ldots \ldots \\
& p q=\mathbf{o d d}, \quad\left\{\gamma_{m_{1} m_{2} \ldots m_{p}}, \gamma^{n_{1} n_{2} \ldots n_{q}}\right\}=\frac{(-1)^{p-1} 2 p ! q !}{1 !(p-1) !(q-1) !} \delta_{\left[m_{1}\right.}^{\left[n_{1}\right.} \gamma_{\left.m_{2} \ldots m_{p}\right]}^{\left.n_{2} \ldots n_{q}\right]}-
\end{aligned}
$$

\footnotetext{
${ }^{2}$ We should note that the coordinate $X^{0} \equiv c t$ is chosen to have length dimensions.
} 


$$
\frac{(-1)^{p-1} 2 p ! q !}{3 !(p-3) !(q-3) !} \delta_{\left[m_{1} m_{2} m_{3}\right.}^{\left[n_{1} n_{2} n_{3}\right.} \gamma_{\left.m_{4} \ldots \ldots m_{p}\right]}^{\left.n_{4} \ldots . n_{q}\right]}+\ldots \ldots
$$

The generalized Kronecker delta is defined as the determinant

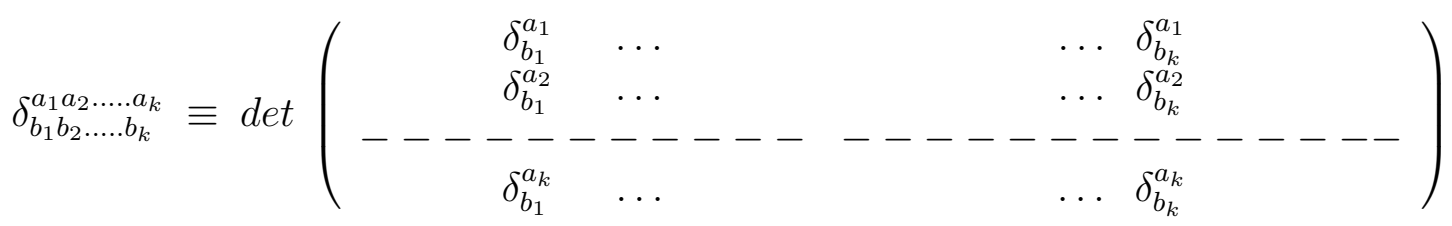

\section{Acknowledgments}

We are indebted to M. Bowers for assistance and to the organizers of the Clifford algebra conference in Tartu, Estonia, for their kind invitation. We thank also Tony Smith, Klee Irwin, Juan Carlos Zambrano, Roberto Lechter and Mohammed Ardehali for their kind support.

\section{References}

[1] C. Castro and M. Pavsic, "The Extended Relativity Theory in Clifford-spaces", Progress in Physics, vol. 1 ( 2005 ) 31. Phys. Letts B 559 (2003) 74. Int. J. Theor. Phys 42 (2003) 1693.

[2] C. Castro, "The Extended Relativity Theory in Clifford Phase Spaces and Modifications of Gravity at the Planck/Hubble scales" , to appear in Advances in Applied Clifford Algebras.

[3] M. Pavsic, Found. of Phys. 33 (2003) 1277.

M. Pavsic, "The Landscape of Theoretical Physics : A Global View, from point particles to the brane world and beyond, in search of a Unifying Principle"” , (Fundamental Theories of Physics, vol. 19, Kluwer Academic Publishers, Dordrecht, Boston, London, 2001).

[4] C. Castro, "Novel Physical Consequences of the Extended Relativity in Clifford Spaces", to appear in Advances in Applied Clifford Algebras.

[5] C. Castro, "Superluminal particles and the Extended Relativity Theories", Foundations of Physics vol 42, issue 9 (2012) 1135.

[6] K. Becker, M. Becker and J. Schwarz, String Theory and M-Theory : An Introduction, pages 543-545 (Cambridge University Press, 2007)

[7] G. Amelino-Camelia, Int. J. Mod. Phys D 11 (2002) 35. Int. J. Mod. Phys D 11 (2002) 1643.

G. Amelino-Camelia, L. Freidel, J. Kowalski-Glikman and L. Smolin, "The principle of relative locality" arXiv.org : 1101.0931. 
[8] C. Castro, "Progress in Clifford Space Gravity", Advances in Applied Clifford Algebras vol 23, Issue 1 (2013). 